\title{
ESTRATÉGIA DE ENFERMAGEM PARA A IMPLEMENTAÇÃO DA ATENÇÃO PRIMÁRIA DE ENFERMAGEM
}

\author{
Nilze Rodrigues Sobreira* \\ Inês Pereira Dantas * \\ Solange Sanchez * \\ Florence Romijn Tocantins *
}

ReBEn/04

SOBREIRA, N.R. e Colaboradoras - Estratégia de Enfermagem para a Implementaçåo da Atenção Primária de Enfermagem. Rev. Bras. Enf.; DF, 34 : 15-34, 1981.

\section{RESUMO}

Os autores tecem considerações sobre as necessidades sanitárias da comunidade brasileira, enfatizando a Política Nacional de Saúde, centrada na ATENÇÃO PRIMÁRIA.

Destacam, ainda, a abrangência dos programas e o posicionamento da Enfermagem para a extensão de cobertura.

Estabelecem, outrossim, uma estratégia de ação, apresentando propostas - alternativas para a implementação de um plano de atuação.

- Professoras da Escola de Enfermagem Alfredo Pinto, do Departamento de Enfermagem Enpecializada - UNI-Rio. 
SOBREIRA, N.R. e Colaboradoras - Estratégia de Enfermagem para a Implementaçāo da Atenção Primária de Enfermagem. Rev. Bras. Enf.; DF, 34 : 15-34, 1981.

\section{CONSIDERAÇÕES GERAIS}

\section{NECESSIDADES SANITÁRIAS}

As necessidades sanitárias da comunidade brasileira caracterizam-se pela magnitude de problemas que se apresentam sob dois aspectos: multiplicidade e extensão.

A multiplicidade pode ser evidenciada por:

- elevada morbi-mortalidade predominantemente no grupo infantil $e$ no grupo inserido na fase economicamente ativa;

- prevalência de grandes endemias como: malária, esquistossomose, doença de Chagas, peste, febre amarela, tracoma, bancroftose, bócio endêmico, tuberculose e hanseníase;

- ocorrência de doenças controláveis por vacinação: a poliomielite, a difteria, o tétano, a coqueluche, o sarampo e a tuberculose;

- desigualdade existente no estado de saúde das comunidades das diversas regiões;

- precariedade de recursos humanos, quer do ponto de vista numérico como iníquo e de distribuição;

- tendência dos serviços a atender somente à demanda, impedindo a antecipação de necessidades de saúde e a solução precoce, refletindo-se numa inversão de assistência eminentemente curativa, em detrimento da assistência preventiva.

Pesa ainda nesta problemática:

- o elevado crescimento demográfico, oscilando em torno de $2,7 \%$ ao ano:

- predominância de tabus culturais, que influenciam negativamente na ação educativa e nas reformas dos programas do Setor Saúde;

- restriçôes de ordem financeira;
- pulverização de recursos, devido à sua inadequada utilização, ocasionando significativa inflação assistencial;

- sistema de informação deficitário, implicando em diagnóstico e programação deficientes;

- defasagem de ordem técnica, institucional ou administrativa entre a Política Nacional de Saúde preconizada e a operacionalização das ações desenvolvidas na realidade;

- deficiência de modelos estruturados de forma operacional, simplificada e adequada às diversas regiões, com estratégias e táticas enfatizadas pela Política Nacional de Saúde.

Toda esta gama de problemas assume um vulto suntuoso, quando vista sob o aspecto EXTENSÃO, podendo ser sintetizada em:

- tendência de propagação das grandes endemias;

- grande demanda assistencial;

- elevados gastos com a assistência;

- repercussões biopsicossocioespirituais sobre o indivíduo, família e comunidade (invalidez, estigma social, conflitos e outros);

- influência nefasta no desenvolvimento sócio-econômico e cultural dos grupos sociais, comunidades e população;

- implicações na produção e, conseqüentemente, na economia;

- persistência de baixos níveis de saúde da comunidade brasileira, causando desperdício econômico e social.

A multiplicidade e a extensão de problemas de saúde concorrem para a renovação da Política Nacional de Saúde.

\section{POLITICA NACIONAL DE SAUDE}

A Política Nacional de Saúde, implícita na Constituição do País, fol instituída pelo Decreto-lei n. ${ }^{\circ} 200$, de 25 de 
SOBREIRA, N.R. e Colaborad.oras - Estratégia de Enfermagem para a Implementação da Atenção Primária de Enfermagem. Rev. Bras. Enf.; DF, 34 : 15-34, 1981.

fevereiro de 1967, visando a definir atribuições dos Governos Federal, Estaduais e Municipais e de Entidades Privadas e estabelecer diretrizes setoriais básicas.

A inobservância desta Política deu origem à organização do Sistema Nacional de Saúde, com o objetivo de disciplinar as ações específicas, conforme a Lei n..$^{\circ} 6.229$, de 17 de julho de 1975.

A institucionalização da Política Nacional de Saúde e do Sistema Nacional de Saúde veio modernizar as ações, até então arcaicas, em virtude do acelerado desenvolvimento sócio-'econômico. Outras modificações vêm ocorrendo, destacando-se, entre elas, a redefinição de alguns termos, estabelecimento de prioridades, simplificação das ações e valorização da participação ativa da comunidade nos programas de saúde.

A magnitude dos problemas e a deficiência de recursos humanos levaram a uma reformulação do conceito de EXTENSÃO DE COBERTURA, até então existente, e ao estabelecimento de programas centrados na satisfação das necessidades básicas do indivíduo, família e comunidade, originando, desta forma, a cognominada ATENÇÃO PRIMÁRIA.

\section{ABRANGÊNCIA DAS AÇÕES}

As ações, outrora decorrentes dos propósitos estabelecidos pelas Instituições, eram estanques e dicotomizadas, predominando aquelas eminentemente curativas ou preventivas, acarretando uma diluição de recursos assistenciais, além do não atendimento às necessidades básicas humanas da comunidade.

A modernização da Política Nacional de Saúde favoreceu, ainda; o atendimento às necessidades básicas de saúde, tanto nos aspectos de promoção e conservação como recuperação e prevenção das doenças, abrangendo, desta forma, os níveis de prevenção PRIMÁRIA, SECUNDARIA e TERCIÁRIA.

\section{POSICIONAMENTO DA ENFERMA- GEM}

Três foram as marcos decisivos para o melhor posicionamento da Enfermagem no Sistema Nacional de Saúde:

- a modernização da Política Nacional:

- a III Reunião Especial de Ministros de Saude das Américas; e

- a varíedade de publicações pertinentes.

Estes fatores vêm contribuindo para uma maior ênfase na A'tenção Primária e para uma redefinição do papel da Enfermagem nesta modalidade de assistência.

Vários pontos têm sido salientados, quanto à Atenção Primária:

- ser um instrumento para a aplicação da Política Nacional de Saúde;

- ser a mesma o ponto-chave para a obtenção da Extensão de Cobertura desejada;

- representar o primeiro nível de contato do indivíduo, família e comunidade com o Sistema Nacional de Saúde.

Não obstante os vários pontos salientados, foram especificadas algumas características essenciais para a implementação da Atenção Primária:

- a utilização de "métodos práticos e científicamente fundamentados e soclalmente aceitáveis";

- o emprego de tecnologia compatível com as características e recursos do individuo, familia e comunidade;

- a participação ativa da comunidade no planejamento e prestação de cuidados;

- baixo custo de operacionalização;

- distribuição eqüitativa da oferta da Atenção Primária;

- adequação à peculiaridade geográfica, financeira, cultural e funcional da comunidade. 
SOBREIRA, N.R. e Colaboradoras - Estratégia de Enfermagem para a Implementação da Atenção Primária de Enfermagem. Rev. Bras. Enf.; DF, 34 : 15-34, 1981.

Ao referir-se à Enfermagem, evidenciam-se alguns aspectos:

- o posicionamento e papel da mesma no Sistema Nacional de Saúde;

- as expectativas quanto à classe e à obtenção da Extensão de Cobertura;

- vantagens e abrangência das ações de Enfermagem.

Todos os fatores, até então revistos, vêm implicando em uma retomada de posição da Enfermagem, levando-a:

- à elaboração de padrões mínimos para a assistência;

- ao estudo e adequação destes padrões às diversas regiões;

-- à reformulação dos planos de assistência;

- ao carreamento de recursos;

- ao treinamento de pessoal e educação continuada;

- à revisão de planos de ensino de Enfermagem nos seus diferentes níveis;

- à integração docente-assistencial.

A revolução na Política Assistencial tem possibilitado uma atuação de Enfermagem mais humana, abrangente, progressiva, dinâmica, metodológica e científica.

\section{ESTRATEGIAS PARA A EFICÁCIA DAS AÇÕES}

As sucessivas mudanças na Política Naclonal Assistencial e as expectativas das autoridades sanitárias quanto à posição da Enfermagem face à Atenção Primária e à Extensão de Cobertura desejada vêm implicando em uma reestruturação, sistematização e elaboração de modelos específicos de Assistência de Enfermagem.

A diversidade de problemas de saúde, as peculiaridades regionais e a variedade de características predominantes dos profissionais e agentes de saúde das diversas áreas motivaram os autores a optarem por estratégias de ações e propostas - alternativas de Atenção Primária de Enfermagem, visando a:

- minimizar a escassez de recursos existentes;

- enfatizar o emprego de procedimentos econômicos;

- estruturar ações efetivas para a definição e elaboração de um subsistema de mecanismo de prestação de serviças de enfermagem;

- assegurar a máxima produtividade e a eficácia dos recursos existentes.

FLUXOGRAMA PARA IMPLEMENTAÇÃO DA ATENÇÃO PRIMÁRIA

Para a implementação da Atenção Primária de Enfermagem, elaborou-se um Fluxograma (Anexú I) onde foram estabelecidas quatro fases consecutivas, consideradas como essenciais: identificação das necessidades da comunidade, elaboração do plano de assistência, implementação do plano e avaliação da Atenção Primária oferecida ao indiví,duo, família e comunidade.

Na primeira fase, considerou-se como instrumento fundamental a realização de um censo ou entrevista, cuja abrangência consiste na verificação das necessidades básicas da comunidade, expectativas dessa comunidade quanto à atenção a ser oferecida, e sua aquiescência na participação dos planos e so-. lução dos problemas que lhes são pertinentes.

A segunda fase envolve uma descrição precisa e global das ações capazes de atender às necessidades identificadas e, ainda, um estudo comparativo entre a Política preconizada e as necessidades reais da comunidade.

O plano deve envolver: objetivos claros, bem definidos e exeqüíveis; metas quantificáveis para a avaliação, definição das atribuições de todos os ele- 
SOBREIRA, N.R. e Colaboradoras - Estratégia de Enfermagem para a Implementação da Atenção Primária de Enfermagem. Rev. Bras. Enf.; DF, 34 : 15-34, 1981.

mentos integrantes lda assistência de Enfermagem; disponibilidade de recursos necessários à implementação da Atenção Primária, estabelecimento de critérios para a avaliação da eficácia da Atenção.

A terceira fase consta da implementação do plano, cujos instrumentos - execução e observação - deverão ser utilizados concomitante e paralelamente, englobando: organização, treinamento de pessoal, modos e meios de participação da comunidade e métodos de utilização de recursos.

A quarta e última fa'se, determinada pela avaliação da Atenção Primária, utiliza os instrumentos - observação e verificação do plano - de forma sincronizada, cuja abrangência consiste num estudo comparativo entre os resultados obtidos e as metas e objetivos preestabelecidos; observação da participação da comunidade em suas diferentes formas (ativa, consciente, responsável, deliberada, organizada e contínua) e a verificação dos níveis de saúde antes da implementação do Plano de Assistência e após a mesma.

\section{PROPOSTAS-ALTERNATIVAS}

Para a implementação e eficácia de um plano de atuação de Enfermagem, inserida no contexto do Sistema Nacional de Saúde e Atenção Primária, optouse pela apresentação de algumas propostais-alternativas (Anexo II), que possam contribuir para a elaboração de modelos de assistência, compatívels com os recursos existentes e com as necessidades reais de cada comunidade.

A utilização das propostas-alternativas implica na observância de alguns princípios essenciais às ações de Enfermagem:

- evitar a desvinculação entre os serviços a serem prestados e as necessidades reais do indivíduo, familia e comunidade;

- atentar para uma melhor utilização dos recursos dentro da escassez e situação real;

- prestar atenção com segurança e eficácia;

- acatar o trabalho interdisciplinar e intersetorial;

- valorizar o indivíduo como um ser psicossomático e a saúde de uma forma integral. 
SOBREIRA, N.R. e Colaboradoras - Estratégia de Enfermagem para a Implementação da Atenção Primária de Enfermagem. Rev. Bras. Enf.; DF, 34 ! 15-34, 1981.

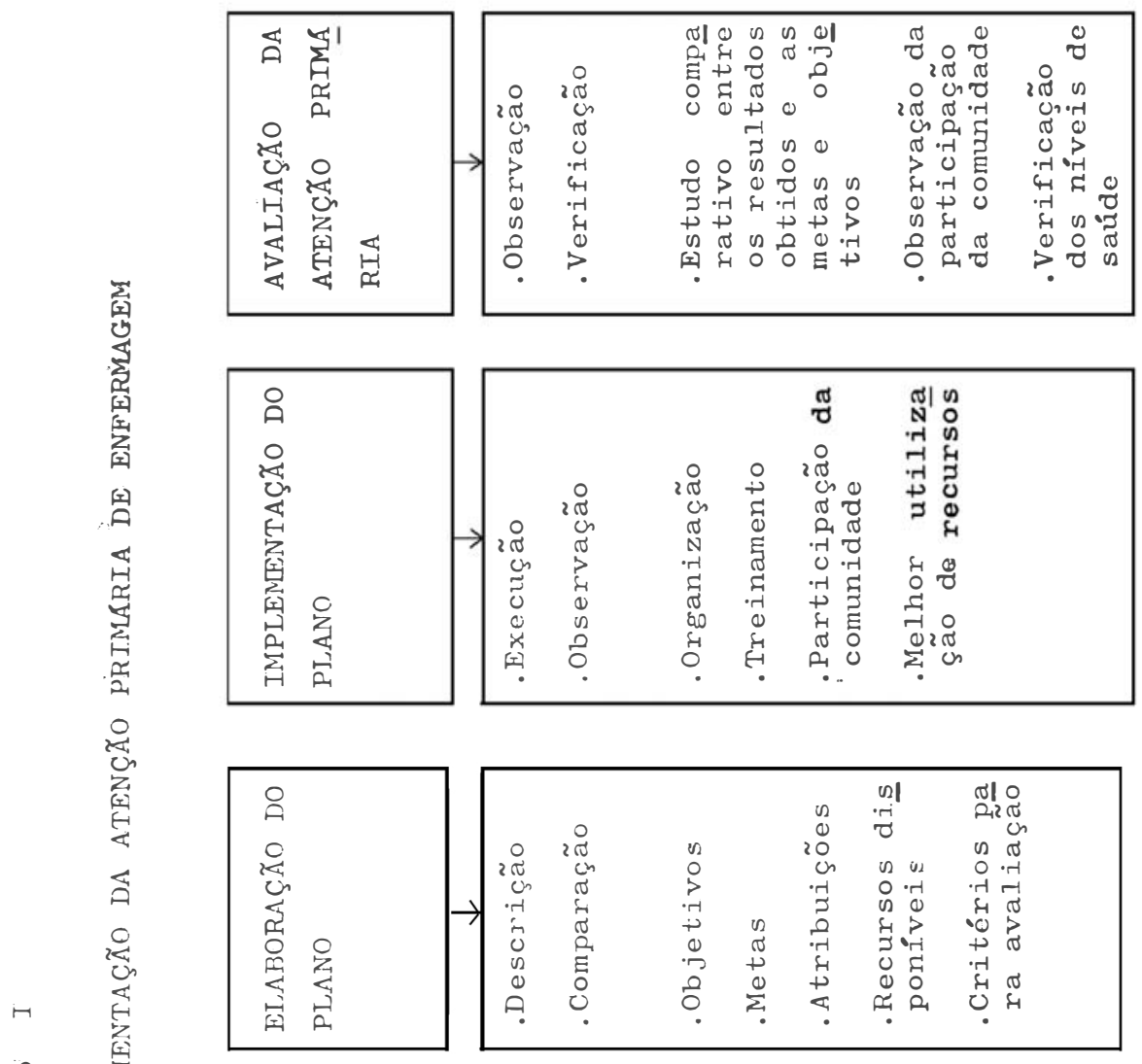

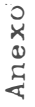

\begin{tabular}{|c|c|c|c|c|c|}
\hline 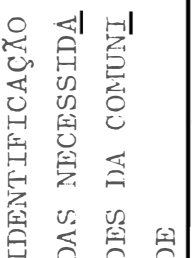 & $\begin{array}{l}0 \\
\text { n } \\
0 \\
0 \\
0\end{array}$ & 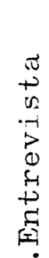 & 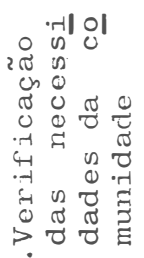 & 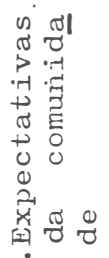 & 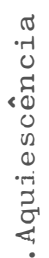 \\
\hline
\end{tabular}

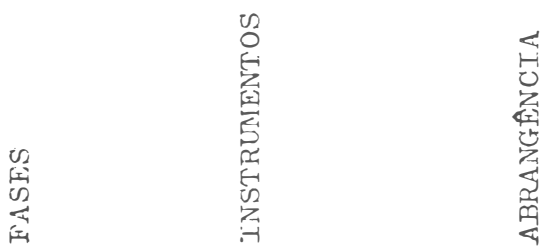


S REIRA, N.R. e Colaboradoras - Estratégia de Enfermagem para a Implementação da Atenção Primária de Enfermagem. Rev. Bras. Enf.; DF, 34 : 15-34, 1981.

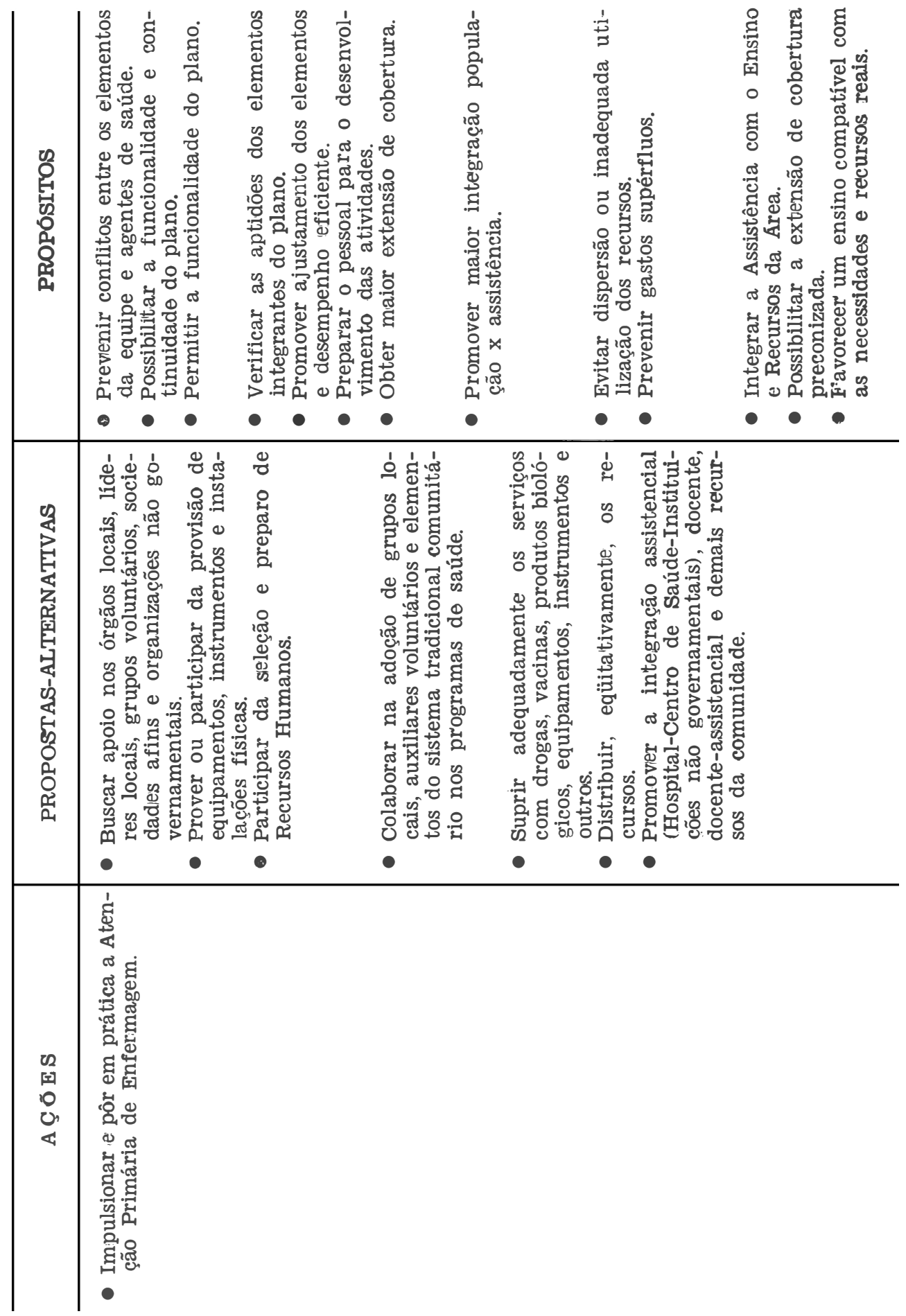


SOBREIRA, N.R. e Colaboradoras - Estratégia de Enfermagem para a Implementação da Atenção Primária de Enfermagem. Rev. Bras. Enf.; DF, 34 : 15-34, 1981.

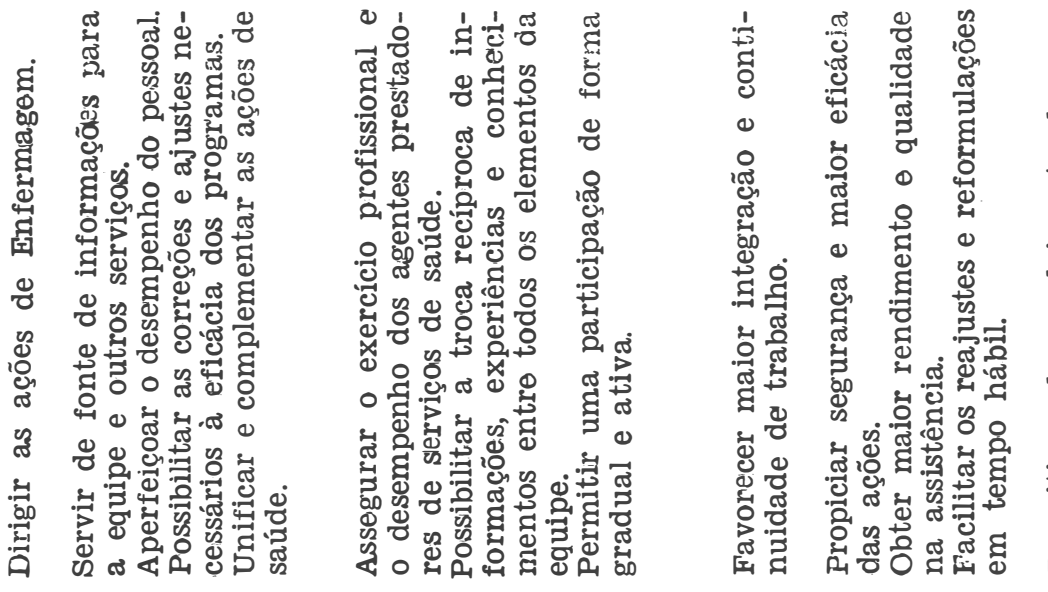

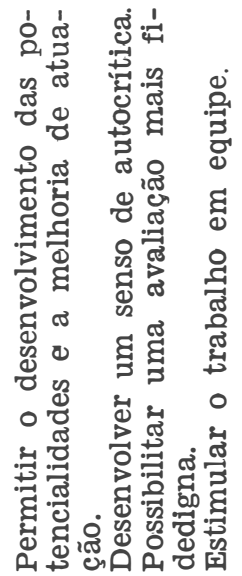

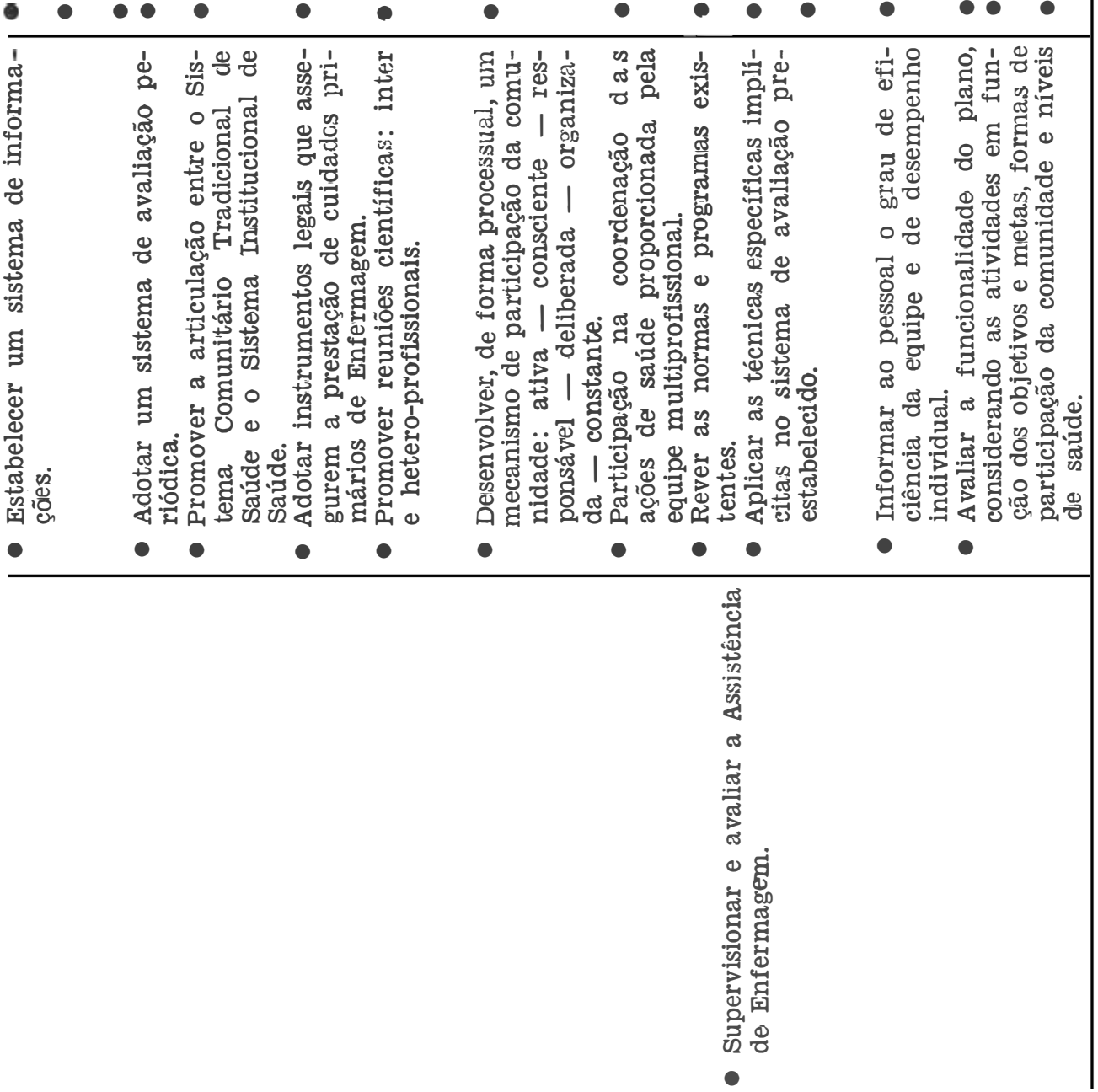


SOBREIRA, N.R. e Colaboradoras - Es'ratégia de Enfermagem para a Implementação da Atenção Primária de Enfermagem. Rev. Bras. Enf.; DF, 34 : 15-34, 1981.

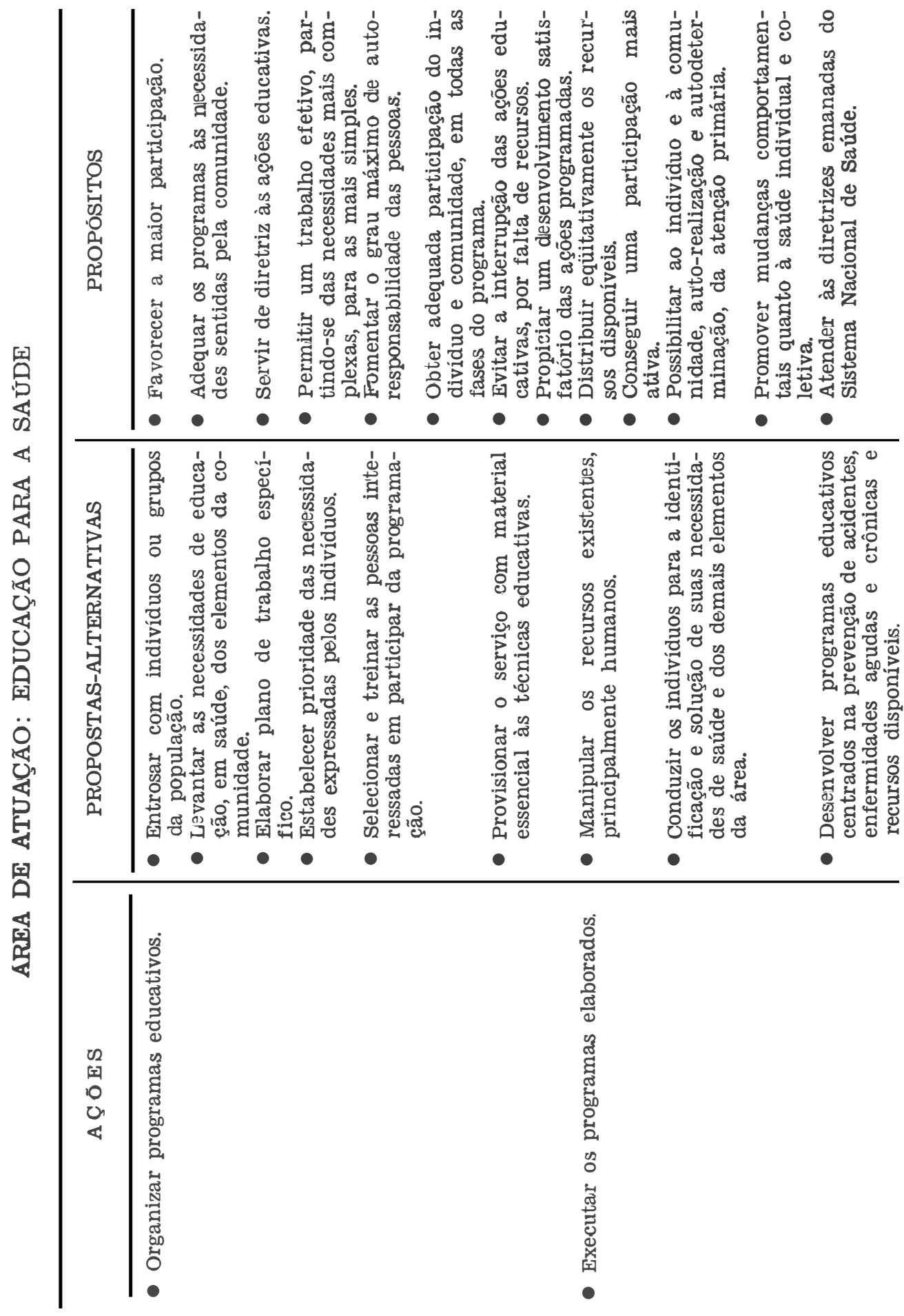




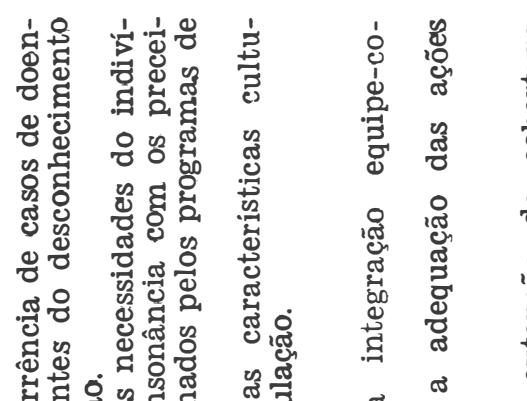

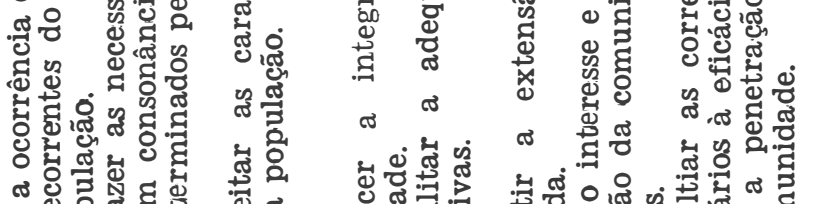

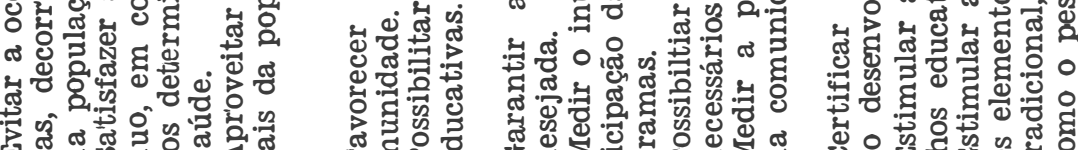

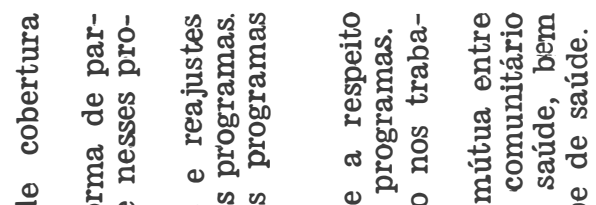

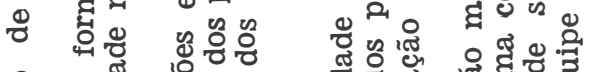
I

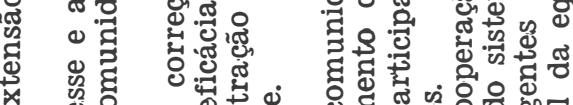

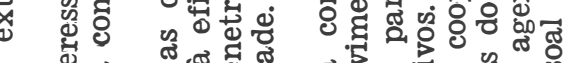
๘

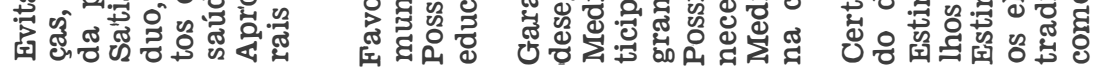

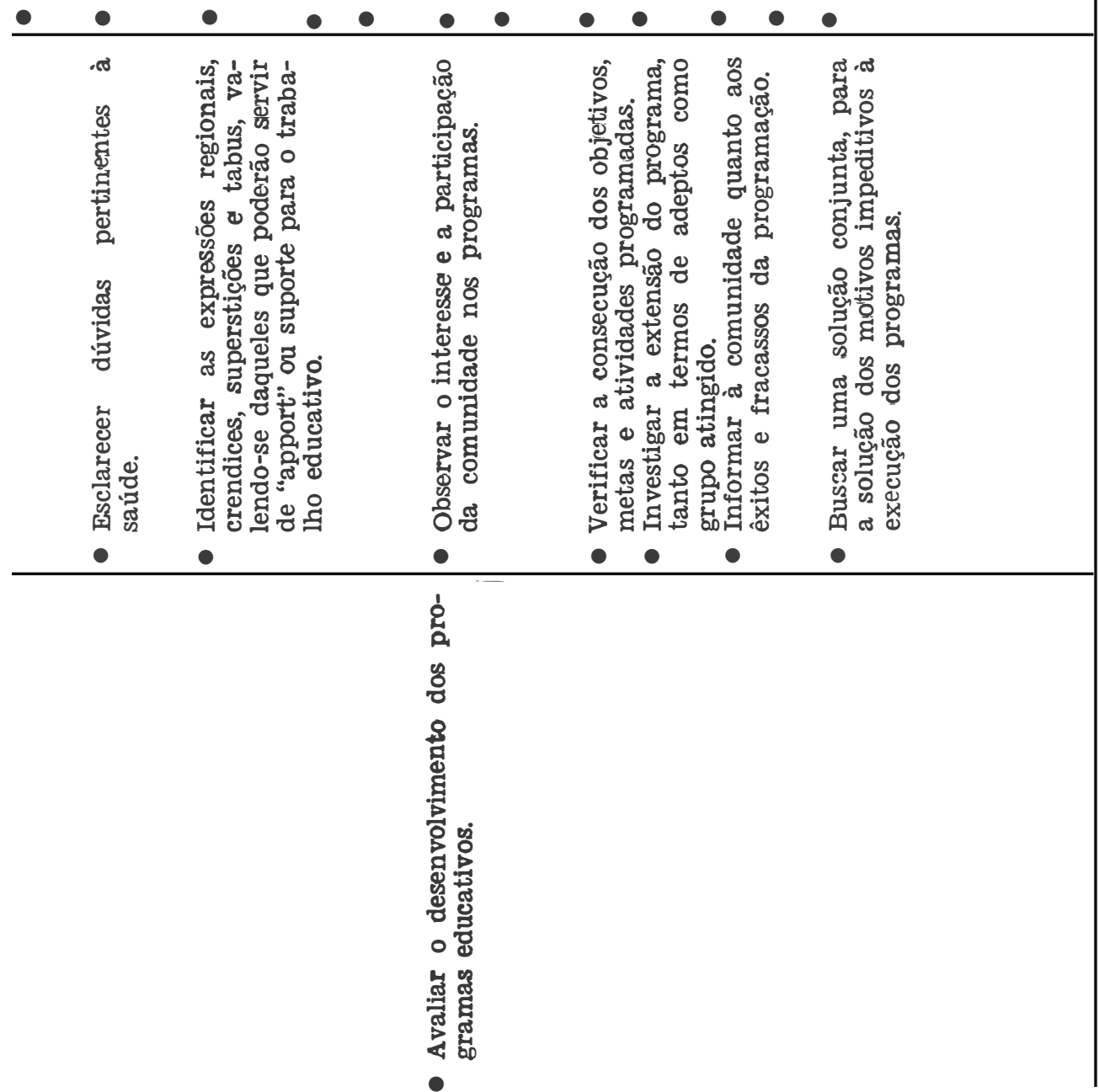


SOBREIRA, N.R. e Colaboradoras - Estra iégia de Enfermagem para a Implementação da Atenção Primária de Enfermagem. Rev. Bras. Enf.; DF, 34 15-34, 1981.

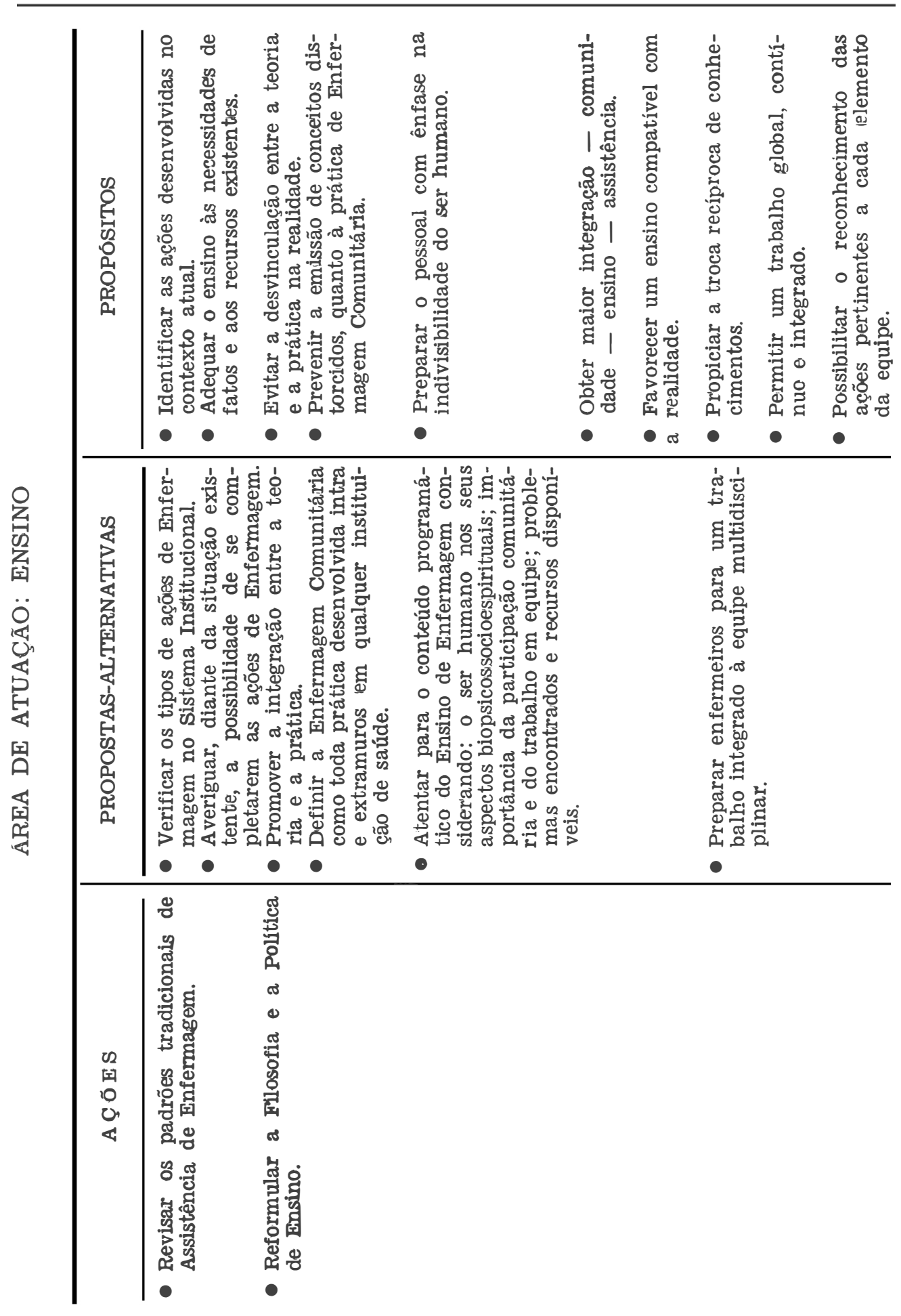


SOBREIRA, N.R. e Colaboradoras - Estratégia de Enfermagem para a Implementação da Atenção Primária de Enfermagem. Rev. Bras. Enf.; DF, 34 : 15-34, 1981.
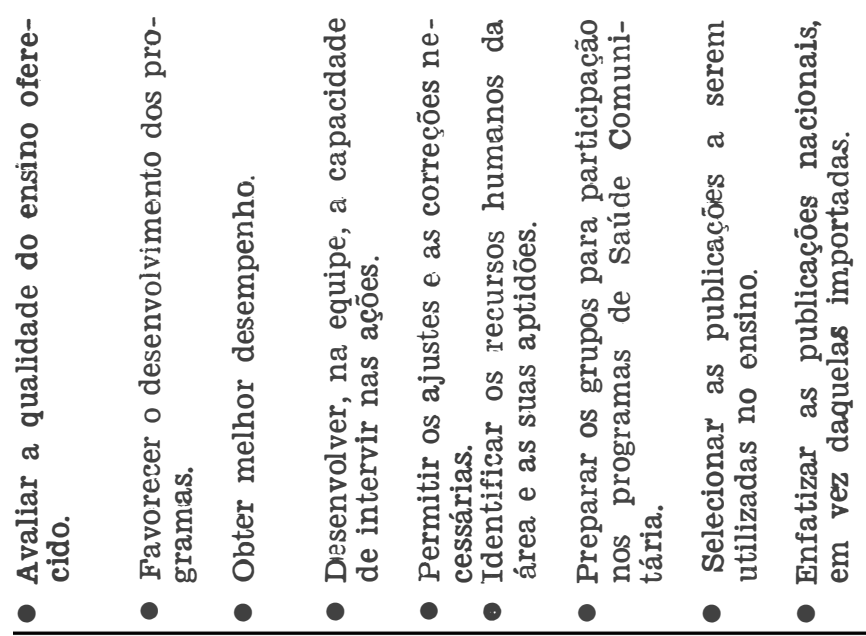

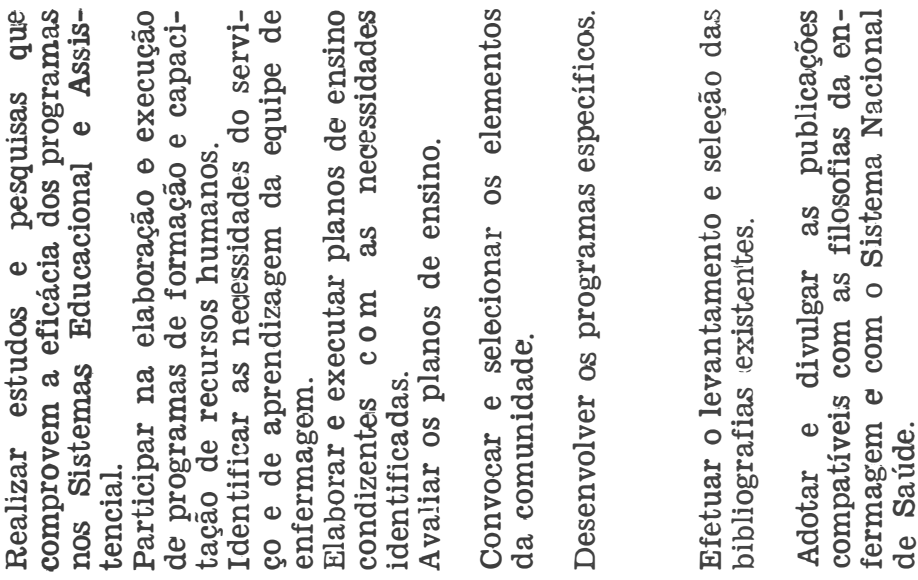

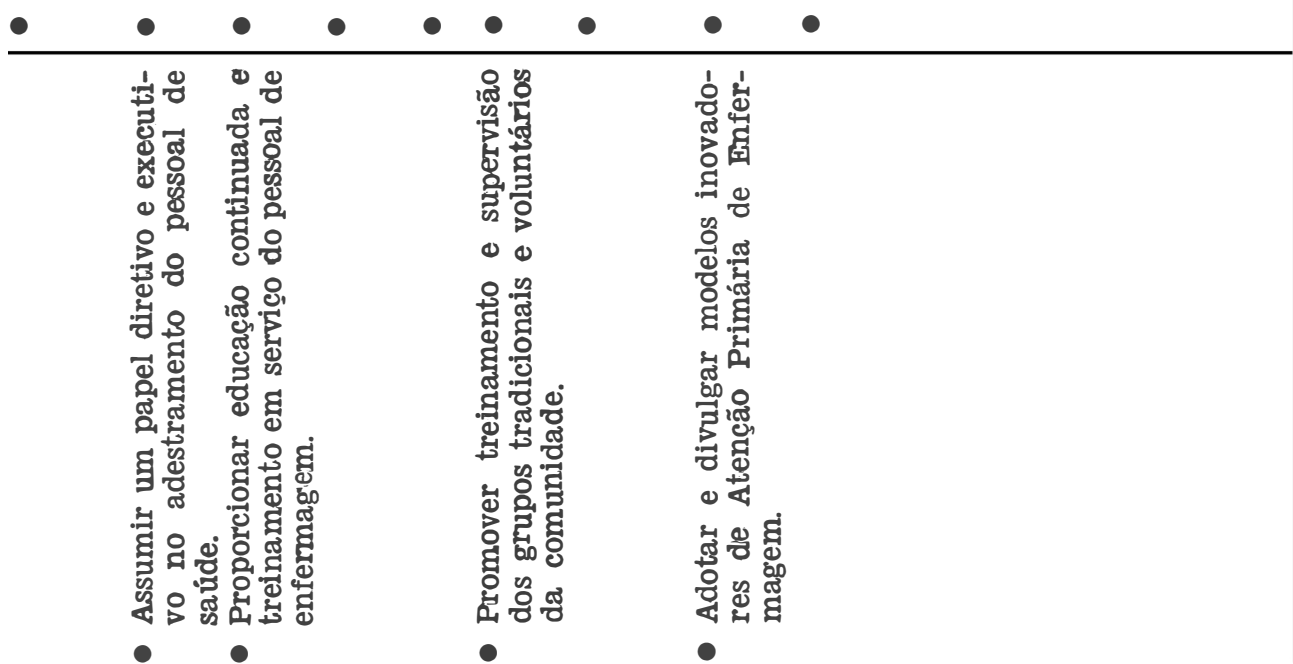




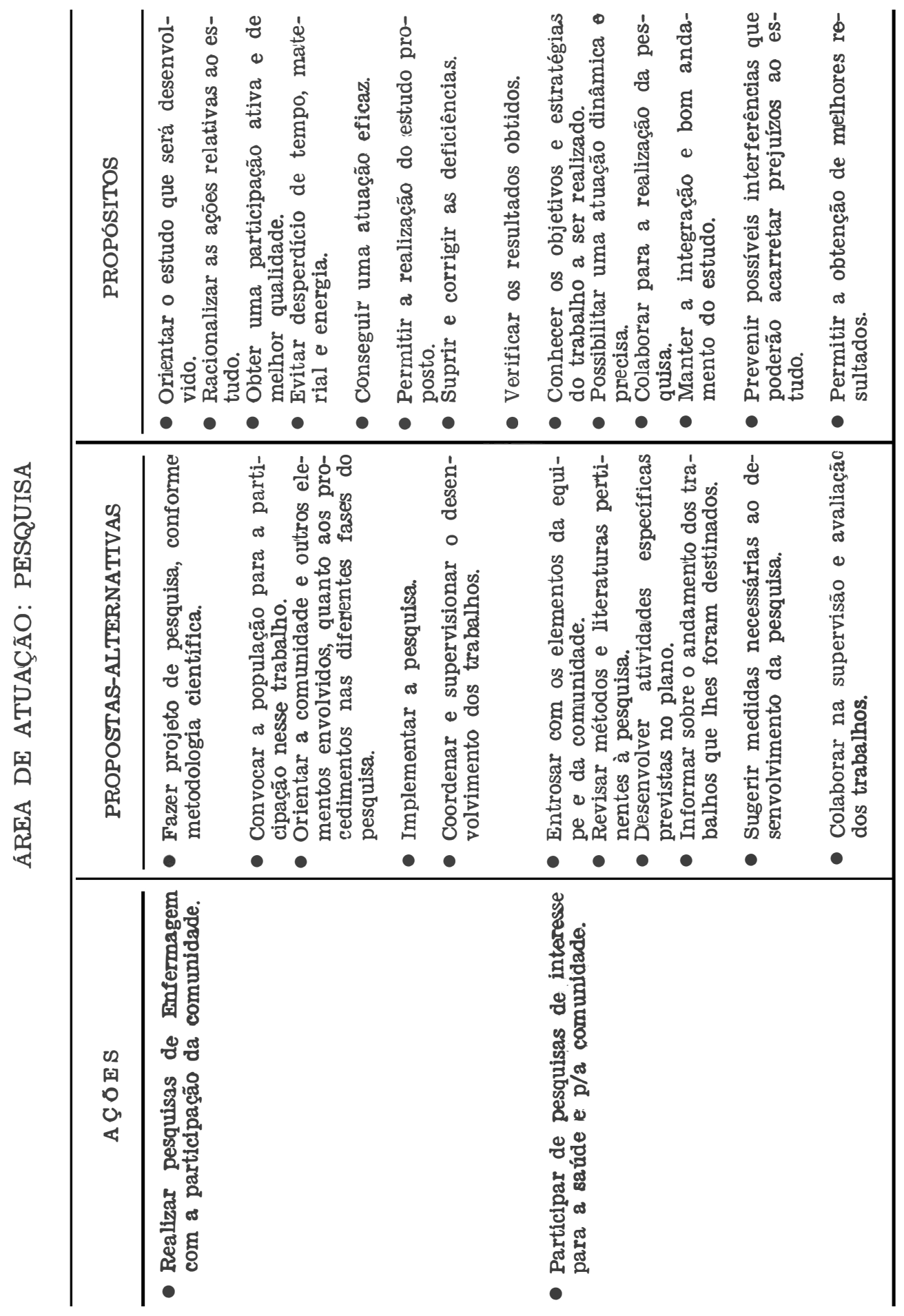


SOBREIRA, N.R. e Colaboradoras - Estratégia de Enfermagem pàra a Implementaçẩo đả Atenção Primária de Enfermagem. Rev. Bras. Enf.; DF, 34 15-34, 1981.

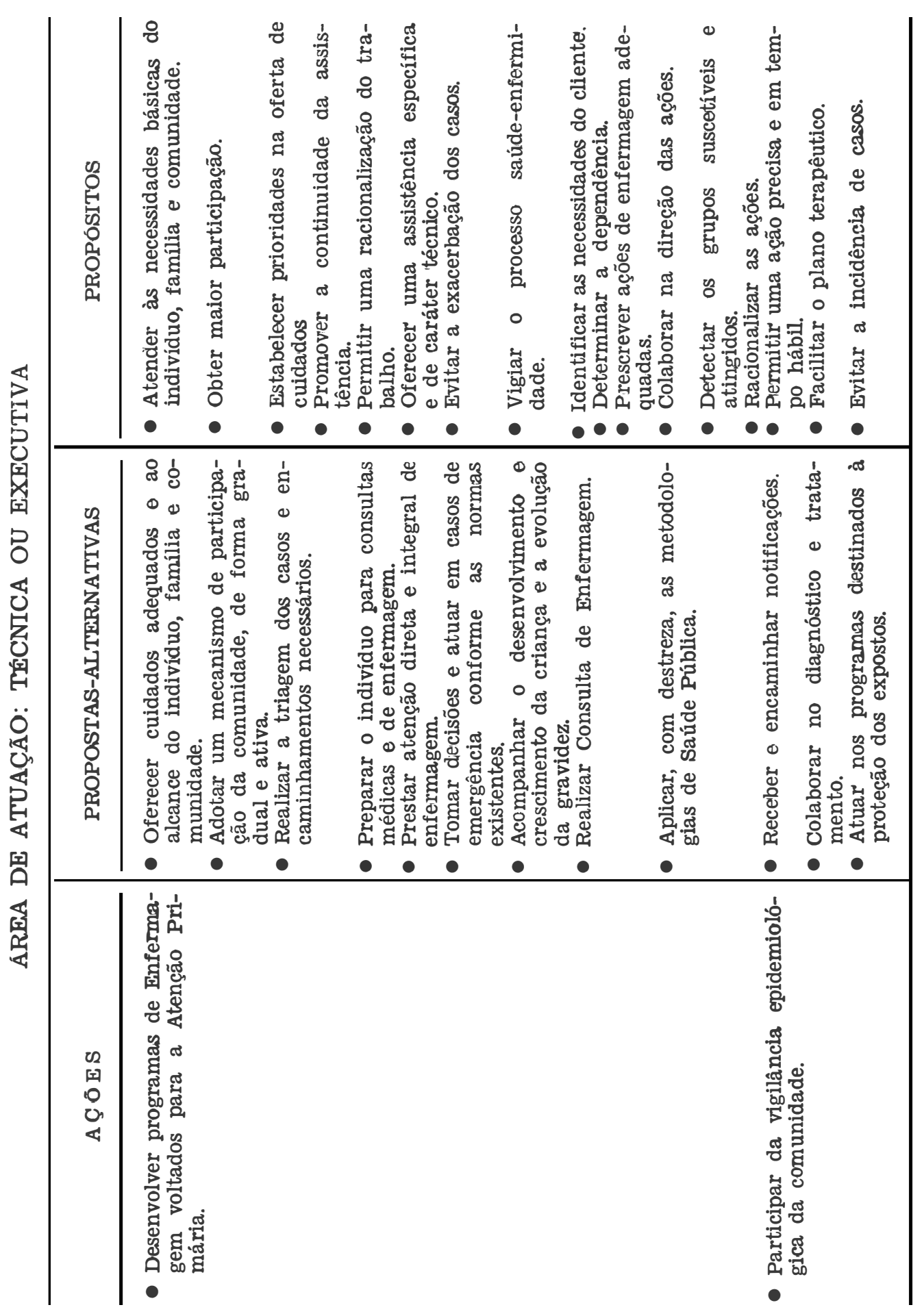


SOBREIRA, N.R. e Colaboradoras - Estratégia de Enfermagem para a Implementaçăo da Atenção Primária de Enfermagem. Rev. Bras. Enf.; DF, 34 : 15-34, 1981.

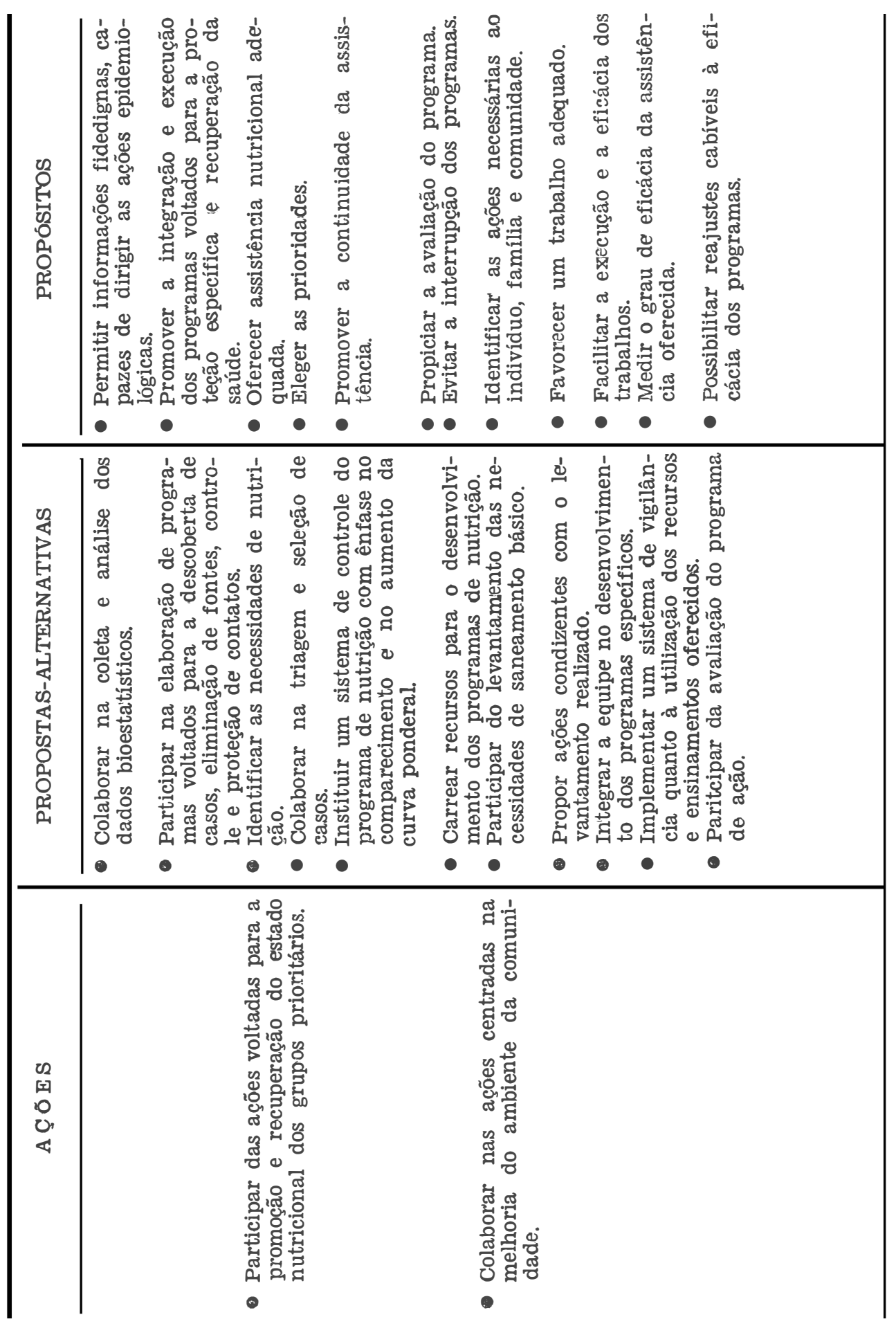




\section{CONCLUSÃO}

A Atenção Primária constitui uma das grandes armas para a integração da comunidade ao Sistema Nacional de Saúde.

O enfoque moderno da participação da comunidade em todas as fases das ações e a reformulação dos conceitos de Assistência e de Exiensão de Cobertura permitem a oferta de atenção mais abrangente, dinâmica e progressiva, coerente com as necessidades, recursos e padrões de vida da comunidade.

A Atenção Primária de Enfermagiem, com respaldo neste novo enfoque e na elaboração de modelos operacionais, possibilita uma ação sistematizada, condizente com os recursos disponiveis e com as expectativas depositadas na Equipe de Enfermagem, pelos Ministros de Saúdie das Américas, e, particularmente, do Brasil.

\section{SUMMARY}

The autors elaborate considerations about the sanitary needs of the Brasilian community, highlighting the Politics of National Health centered upon the Primary Health Care.

They consider of significant 1mportance the extension of the programs as well as the nurse's position in the process.

The autors establish, therefore, strategic ways and alternatives for the fullfilment and efficacy of a working plan.

\section{TERMINOLOGIA}

Os termos empregados neste trabalho podem ser definidos como:

\begin{tabular}{|c|c|}
\hline TERMOS & D E F I N I Ç Ã O \\
\hline - ACESSIBILIDADE FINANCEIRA & $\begin{array}{l}\text { ou seja, os pagamentos ou contribui- } \\
\text { ções para utilização dos serviços não } \\
\text { devem constituir obstáculos. }\end{array}$ \\
\hline - ACESSIBILIDADE CULTURAL & $\begin{array}{l}\text { ou seja, não deve haver conflito entre } \\
\text { os padrões técnicos e administrativos } \\
\text { dos serviços e os hábitos, padrões cul- } \\
\text { turais e costumes das comunidades em } \\
\text { que sejam prestados. }\end{array}$ \\
\hline - ACESSIBILIDADE GEOGRAFICA & $\begin{array}{l}\text { ou seja, a distância, o tempo de loco- } \\
\text { moção e os meios de transporte devem } \\
\text { determinar a localização dos estabele- } \\
\text { cimentos e não áreas teóricas de juris- } \\
\text { dição. }\end{array}$ \\
\hline - ACESSIBILIDADE FUNCIONAL & $\begin{array}{l}\text { ou seja, os servicos devem ser presta- } \\
\text { dos oportunamente e em caráter con- } \\
\text { tínuo, bem como estar disponíveis em } \\
\text { qualquer momen'to, atender à deman- } \\
\text { da real e incluir um Sistema die refe- } \\
\text { rência que assegure fácil acesso ao ní- } \\
\text { vel de assistência que se requer. }\end{array}$ \\
\hline - ATENÇÃO PRIMÃRIA & $\begin{array}{l}\text { * conjunto de acões que visam a } \\
\text { satisfazer as necessidades básicas de }\end{array}$ \\
\hline
\end{tabular}


SOBREIRA, N.R. e Colaboradoras - Estratégia de Enfermagem para a Implementação da Atenção Primária de Enfermagem. Rev. Bras. Enf.; DF, 34 : 15-34, 1981.

\begin{tabular}{l|l}
\hline \multicolumn{1}{c|}{ TERMOS } & \multicolumn{1}{c}{ D E F I N T Ç Ã O } \\
\hline \multirow{2}{*}{ COBERTURA } & $\begin{array}{l}\text { saúde, tanto nos aspectos de promoção } \\
\text { e conservação como recuperação e pre- } \\
\text { venção de enfermidadies. }\end{array}$ \\
& $\begin{array}{l}\text { E resultado de uma oferta eficaz e } \\
\text { organizada de Serviços Básicos de Saú- } \\
\text { de que atendam às necessidades de } \\
\text { toda população, continuamente presta- } \\
\text { dos em locais a que ela tem acesso e } \\
\text { da maneira que mais lhe convenha, as- } \\
\text { segurando-lhe o acesso, também, dos } \\
\text { diferentes níveís de assistência do Sis- } \\
\text { tema de Serviços de Saúde. }\end{array}$ \\
\hline
\end{tabular}

- CUIDADOS PRIMÁRIOS

- COMUNIDADE

- OUTROS NÍVEIS DE ASSISTÊNCIA

- PARTICIPAÇÃo COMUNITÁRIA

- participação ativa

PARTICIPAÇÃO CONSCIENTE

- participação contínUa

PARTICIPAÇÃo DELIBERADA

PARTICIPAC̣̃o ORGANIZAda
Entende-se a acessibilidade de todos os indivíduos e famílias de uma comunidade a serviços essenciais de saúde prestados por meios que lhes sejam aceitáveis, através de sua participação integral e a custo que a comunidade e o país possam absorver.

É um grupo social determinado por limites geográficos e/ou valorles e interesses comuns.

Representam serviços especializados, de crescente complexidade que, em geral, funcionam mediante referências individuais.

f o processo pelo qual indivíduos e famílias assumem a responsabilidade pela saúde e o bem-estar próprios e da comunidade, e desenvolve a capacidade de contribuição para o desenvolvimento pessoal e comunitário.

É quando os indivíduos intervêm nas várias etapas do processo.

É quando os indivíduos en'tendem plenamente os problemas, traduzem-nos em necessidades palpáveis e trabalham para a sua solução.

É quando os indivíduos atuam permanentemente em conjunto para a solução dos vários problemas da comunidade.

É quando os indivíduos expressam sua livre vontade.

É quando os indivíduos percebem a necessidade de conjugar seus esforços no sentido de objetivos comuns. 
SOBREIRA, N.R. e Colaboradoras - Estratégia de Enfermagem para a Implementação da Atenção Primária de Enfermagem. Rev. Bras. Enf.; DF, 34 : 15-34, 1981.

\begin{tabular}{|c|c|}
\hline T E R MOS & D E F I N I Ç Ã O \\
\hline - PARTICIPAÇÃO RESPONSÁVEL & $\begin{array}{l}\text { E quando os indivíduos se comprome- } \\
\text { tem e decidem agir com plena cons- } \\
\text { ciência de suas obrigações e das con- } \\
\text { seqüências destas. }\end{array}$ \\
\hline - POLfTICA NACIONAL DE SAÚdE & É um conjunto de princípios e diretri- \\
\hline
\end{tabular}
zes destinados a orientar a ação e decisão do governo no setor saúde.

- PREVENÇÃo

É a aplicação da tecnologia e de métodos científicos, a nível de grupos ou indivíduos, para promover, manter ou restaurar a saúde.

- PRIMEIRO NIVEL DE ASSistencia

É a modalidade de assistência que inclui os elementos mais elementares e menos diferenciados do Sistema e constitui o ponto de contato com a comunidade e seu meio de acesso ao sistema institucional.

F. o resultado de uma relação recíproca cio homem com o seu ambiente.

- SAÚde Da COMUNIDAde

- sistema comUnitário tRadiCIONAL

É aquele estabelecido pela própria comunidade e usado pelos seus membros, que recorrem à automedicação ou a algum membro da comunidade reconhecido como representante do sistema.

- sistema institucional DE SAÚDE

É aquele constituído de instituições públicas e privadas de saúde.

E um mecanismo pelo qual os recursos e a capacidade instalada se organizam através de estrutura administrative $\mathrm{e}$ de uma tecnologia médica para oferecer prestação de Serviços de Saúde integrados, em quantidade suficien's e quantidade adequada, para cobrir a demanda da comunidade, a um custo compativel com os recursos financeiros disponiveis. 
SOBREIRA, N.R. e Colaboradoras - Estratégia de Enfermagem para a Implementação da Atenção Primária de Enfermagem. Rev. Bras. Enf.; DF, 34 : 15-34, 1981.

\section{B I B L I O G R A F I A}

1. COSTA, Z. S.; SOBREIRA, N. R.; LEITE, J. L.; DANTAS, I. P. - A Enfermagem Comunitária e o Sistema Nacional de Saúde Trabalho apresentado em Congresso, Belém, 1978.

2. DANTAS, I. P. - A Enfermagem de Saúde Pública na Assistência Materno-Infantil - Editora JUERP, Rio de Janeiro, 1978.

3. OMS/UNICEF - Cuidados primários de Saúde - Alma-Ata, 1978.

4. OPS/OMS - Extensão de cobertura dos Serviços de Saúde mediante o uso das estratégias de Assistência Pri- mária e participação da comunidade - Washington, D.C., 1977.

5. OPS/OMS - Enseñanza de Enfermeria en Salud Comunitaria, Washington, D.C., 1975.

6. SAÚD王, Ministério da - Política Nacional de Saúde - Ministério da Saúde, Brasília, D.F., 1973.

7. SAÚdE, Ministério da - VI Conferência Nacional de Saúde - Ministério da Saúde, Brasília, D.F., 1977.

8. SOBREIRA, N. R. - Enfermagem Comunitária - no prelo - Rio de Janeiro, 1980. 